\title{
Photovoltaic performances of mono- and mixed-halide structures for perovskite solar cell: a review
}

\begin{abstract}
This review discusses the photovoltaic performances of mono- and mixed-halide perovskite solar cells, which include the band gap, absorption spectrum, hysteretic behavior, impedance spectroscopy results, and current-voltage measurements. It is desirable to obtain a highperformance perovskite material for use as a light harvester with excellent photovoltaic performances, including a high open circuit voltage (Voc), high short circuit current density (Jsc), high efficiency with minor recombination rate, and large optical absorption ability. In addition, this perovskite material should be able to harvest light over the entire absorption spectrum. Typically, the near-IR region is highly favorable for obtaining the highest power conversion efficiency (PCE) for a solar cell. However, to date, the optical absorption ability of a lead-based single halide perovskite solar cell is still constrained below the near-IR region, which hinders its performance as a fully efficient perovskite solar cell. The insertion of tin within the methylammonium lead halide matrix, which forms $\mathrm{CH} 3 \mathrm{NH} 3 \mathrm{SnxPb}(1-\mathrm{x}) \mathrm{I} 3$, provided excellent light absorption with photo-response coverage up to $1060 \mathrm{~nm}$. Nevertheless, the low efficiency and low Voc of the tin halide-based perovskite solar cell ascribed to the instability of the Sn (II) ion hinders the current solar cell application despite its good lightharvesting performance. Another approach to enhance the photovoltaic performance involves tailoring mixed halides for a perovskite solar cell. A wider absorption spectrum range is obtainable, and the band gap energy of the perovskite solar cell is tunable by adjusting the ratio of the mixed halides. The perovskite solar cells have high potential to be the largest energy production in the near future owing to their low module cost with high conversion efficiency, comparable to the silicon solar cell.
\end{abstract}

Keyword: Perovskite; Efficiency; Band gap; Absorption spectrum; Hysteretic; Impedance and stability 